\title{
Awake Surgical Mitral Valve Repair after Transcatheter Aortic Valve Replacement
}

\author{
Aina Hirofuji ${ }^{1}$ Hirotsugu Kanda ${ }^{2}$ Yuya Kitani $^{3} \quad$ Hiroyuki Kamiya $^{1}$ \\ ${ }^{1}$ Department of Cardiac Surgery, Asahikawa Medical University, \\ Asahikawa, Japan \\ 2 Department of Anesthesiology, Asahikawa Medical University, \\ Asahikawa, Japan \\ 3 Department of Cardiology, Asahikawa Medical University, \\ Asahikawa, Japan \\ Address for correspondence Hiroyuki Kamiya, MD, PhD, Department of \\ Cardiac Surgery, Asahikawa Medical University, Midorigaoka-Higashi, \\ Asahikawa 078-8510, Japan (e-mail: hkamiya88@yahoo.co.jp). \\ Thorac Cardiovasc Surg Rep 2021;10:e15-e17.
}
Abstract Keywords
- aortic stenosis
- mitral regurgitation
- transcatheter aortic valve replacement
- minimally invasive cardiac surgery

Transcatheter aortic valve replacement has become a popular choice for cases with severe aortic stenosis. However, when severe mitral regurgitation is comorbid in highrisk patients with severe aortic stenosis, therapeutic options must be weighed for each case. Here we present a very frail 88-year-old patient with severe aortic stenosis and severe mitral valve regurgitation who underwent a successful awake minimally invasive mitral valve repair after transcatheter aortic valve replacement.

\section{Introduction}

With evolving transcatheter technologies, many new and safe therapeutic options for multiple valvular heart disease (VHD) have become available. However, treatment strategies for very high-risk cases remain controversial. Here we present a very frail 88-year-old patient with severe aortic stenosis (AS) and severe mitral valve regurgitation (MR) who underwent a successful awake minimally invasive mitral valve repair (MIMVS) after transcatheter aortic valve replacement (TAVR).

\section{Case Report}

Submission and publication of this case report was approved by the institutional review board (No. 19207) and a written informed consent was obtained from the patient.

An 88-year-old female (height, $141 \mathrm{~cm}$; weight, $34 \mathrm{~kg}$ ) with a history of chronic heart failure due to severe AS and severe MR presented with loss of appetite and shortness of breath. Transthoracic and transesophageal echocardiograms showed a tricuspid aortic valve with a peak velocity of $4.1 \mathrm{~m} / \mathrm{s}$, mean pressure gradient of $38 \mathrm{mmHg}$, and valve area of $0.52 \mathrm{~cm}^{2}$. The MR was caused by P2 prolapse (- Figs. 1 and $\mathbf{2 A}$ ), and consisted of three regurgitant jets, and the vena contracta was $8.1 \mathrm{~mm}$. There was also moderate mitral annular calcification. Although the severity of her heart failure and VHD were indications for surgery, due to the patient's age, high frailty scored 7.5 on the Clinical Frailty Score, and general poor condition, the risks of open heart surgery calculated on the JapanSCORE were $16.3 \%$ for mortality, while the STS score was 4.55\%. Our heart team decided on TAVR for primary treatment in hopes that treating the severe AS would improve the MR and heart failure. If post-TAVR course proved inadequate after ample time and observation, we would go on to perform mitral valve repair.

TAVR was performed under local anesthesia (total dose: $20 \mathrm{~mL}$ of $1 \%$ lidocaine with epinephrine) in the transfemoral approach with a 26-mm SAPIEN 3 device (Edwards Life Science, Irvine, California, United States). Throughout the intervention, the patient was lightly sedated using targetcontrolled intravenous infusion of propofol $(0.1-0.7 \mu \mathrm{g} / \mathrm{mL})$ and continuous infusion of dexmedetomidine $(0.7 \mu \mathrm{g} / \mathrm{kg} / \mathrm{h})$. received

June 30,2020

accepted

August 21, 2020
DOI https://doi.org/

10.1055/s-0040-1718774. ISSN 2194-7635.

\footnotetext{
(C) 2021. The Author(s).

This is an open access article published by Thieme under the terms of the Creative Commons Attribution-NonDerivative-NonCommercial-License, permitting copying and reproduction so long as the original work is given appropriate credit. Contents may not be used for commercial purposes, or adapted, remixed, transformed or built upon. (https://creativecommons.org/ licenses/by-nc-nd/4.0/) Georg Thieme Verlag KG, Rüdigerstraße 14, 70469 Stuttgart, Germany
} 

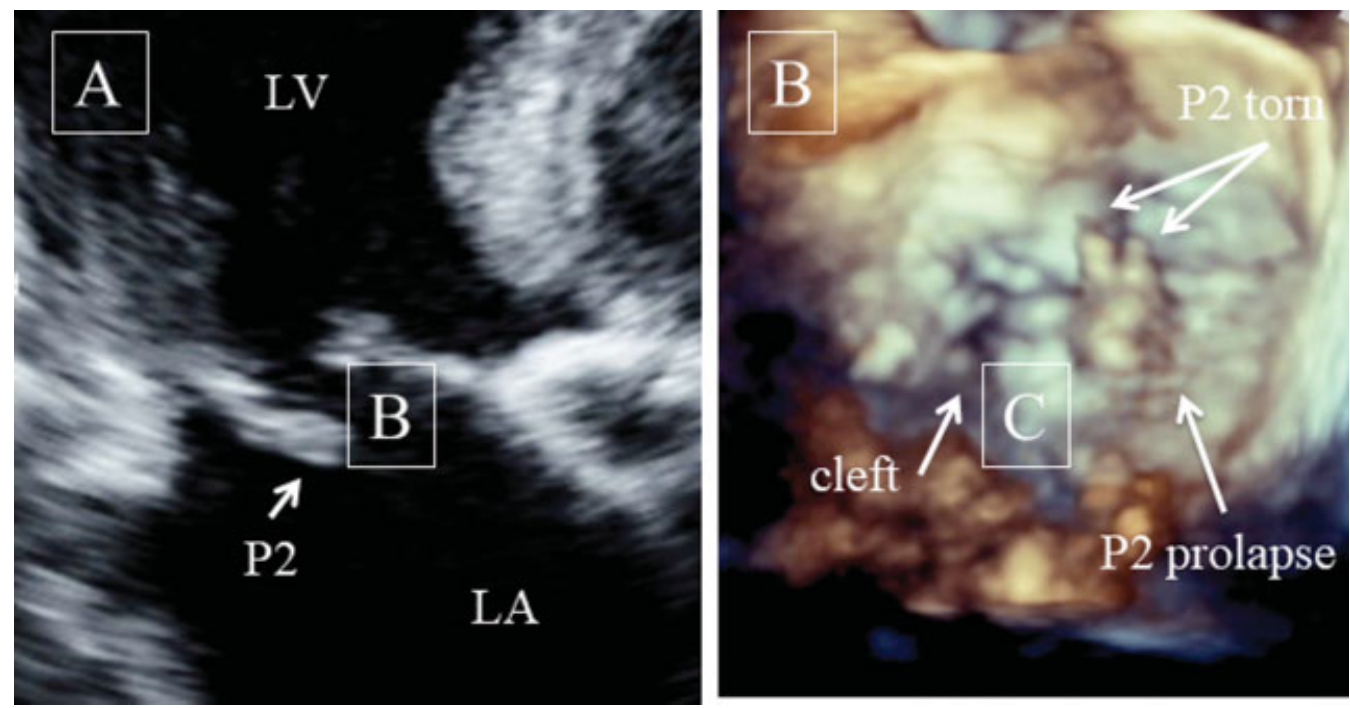

Fig. 1 Initial transthoracic (A) and transesophageal (B) echocardiograms showed mitral valve regurgitation (MR) caused by P2 prolapse due to torn chordae tendineae.
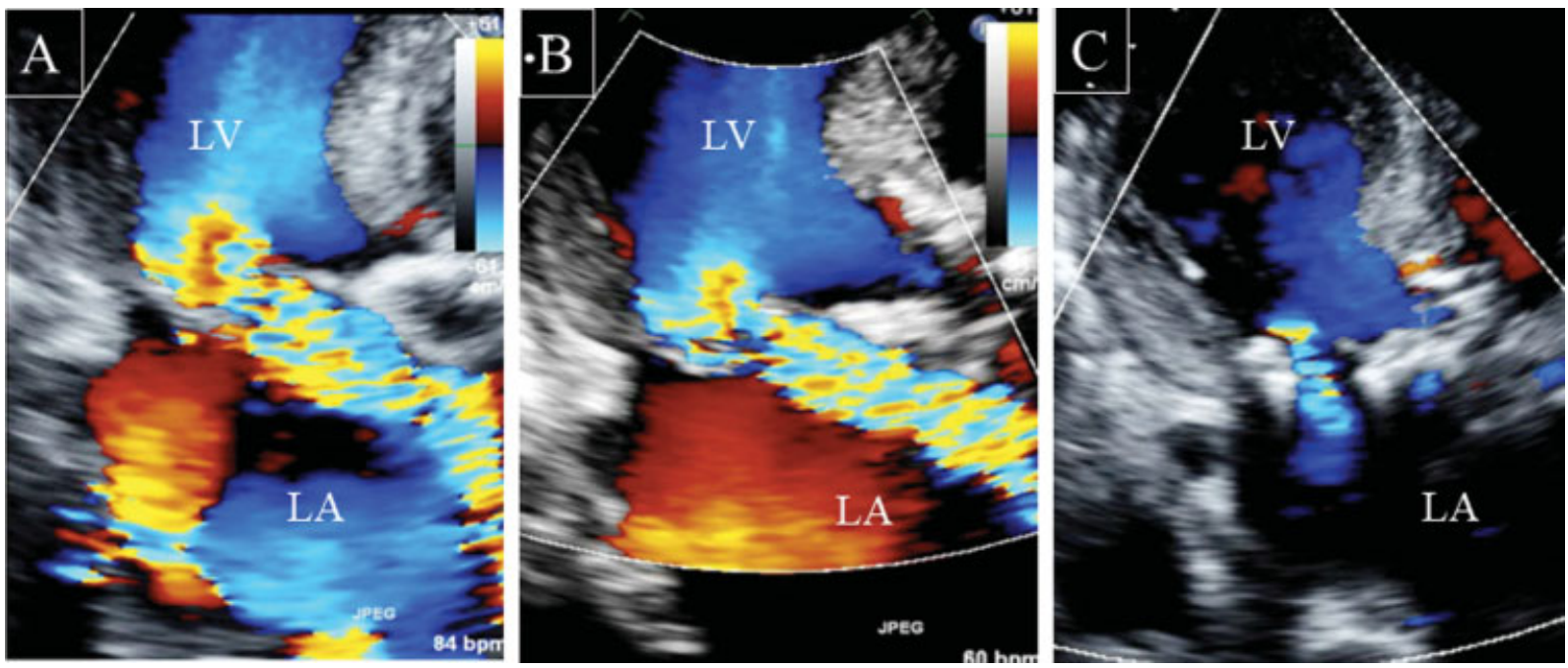

Fig. 2 Pre-transcatheter aortic valve replacement (TAVR), transthoracic echocardiogram (TTE) showed a severe eccentric mitral valve regurgitation (MR) jet in the anterior medial direction (A). Post-TAVR, MR severity remained unchanged (B). Post-mitral valve repair by minimally invasive cardiac surgery (MICS), the severity of MR was greatly reduced (C).

The post-TAVR echocardiogram showed improvement of AS; however, the MR remained severe without any significant change or improvement (-Fig. 2B). Also, the patient's heart failure remained difficult to manage with medication alone, with little to no improvement in her dyspnea or appetite.

In our initial heart team conferences, we had concluded that treating the MR with MitraClip (Abbott, Illinois, United States) would be contraindicative because it would require multiple clips, and the risk of causing severe mitral stenosis due to the highly calcified mitral valve annulus. Therefore, we decided on MIMVS via right minithoracotomy for the patient. Considering her highly frail condition, this operation was done avoiding general anesthesia, and on day 26 post-TAVR, minimally invasive cardiac surgery (MICS) was performed. In the operating room, the patient was sedated with target-controlled intravenous infusion of fentanyl $(0.1-1.0 \mu \mathrm{g} / \mathrm{mL})$ and continuous infusion of dexmedetomidine $(0.7 \mu \mathrm{g} / \mathrm{kg} / \mathrm{h})$. Before surgical intervention, the maximum total dose $0.25 \%$ levobupivacaine $40 \mathrm{~mL}$ ) was injected at the incision site to reduce surgical pain and body movement. After initiation of cardiopulmonary bypass (CPB) and right-sided thoracotomy, the $\mathrm{PaCO}_{2}$ level was kept at approximately $30 \mathrm{~mm} \mathrm{Hg}$, providing intentional apnea and a clear operative field. Regular MICS retraction devices were used. The mitral valve was exposed slowly and carefully with a retractor as not to cause any TAVR valve migration by avoiding the left atrial area where the TAVR valve was pushing out on, then the retractor was fixed securely once sufficient operation field was attained. Mitral valve repair with P2 triangular resection and annuloplasty with Future-Band $28 \mathrm{~mm}$ (Medtronic, Minneapolis, Minnesota, United States) were successfully performed as usual ( $\mathbf{- V i d e o} \mathbf{1}$ ). Before the termination of $\mathrm{CPB}$, the $\mathrm{PaCO}_{2}$ level was maintained at approximately $50 \mathrm{~mm} \mathrm{Hg}$ to restart spontaneous ventilation. 
Aortic clamping time, CPB time, and operation times were 56, 127 , and 164 minutes, respectively. During surgery, the patient felt no pain and minimal discomfort; the anesthesiologist regularly checked with the patient through verbal and gestural communication. Her postoperative course was uncomplicated, and echocardiogram showed mild residual MR (-Fig. 2C), but her heart failure had greatly improved. The patient was discharged to a rehabilitation hospital on postoperative day 11.

\section{Video 1}

Frame from video. Sufficient surgical field was attained with a fixed retractor. Retraction was done carefully as not to cause migration of the transcatheter aortic valve replacement (TAVR) valve pushing out into the left atrium (LA). Online content including video sequences viewable at: https://www.thieme-connect.com/ products/ejournals/html/10.1055/s-0040-1718774.

\section{Comment}

Severe MR often coexists with severe AS, and the best treatment strategy is sometimes unclear, especially in elderly high-risk patients because multiple valve operations are significantly linked to higher morbidity and mortality especially in the elderly. ${ }^{1}$ There have been yet no guidelines for such high-risk cases, therefore the management of elderly patients with coexisting severe AS and MR is left to the primary medical team.

Considering the risk factors for conventional double valve repair, we decided to proceed with TAVR, because it has been reported that MR grade improves post-TAVR in 25 to $50 \%$ of cases. ${ }^{2}$ If there was improvement of the MR with only TAVR, it would be the least invasive outcome. According to Sannino and Grayburn's comprehensive retrospective study of coexisting AS and MR cases, factors that decrease and increase MR severity post-TAVR were mixed in this particular case. However, in their algorithm for management of concomitant AS and MR cases, they propose TAVR followed by minimally invasive or transcatheter MV surgery. ${ }^{3}$ In the present case, the treatment course with TVAR followed by awake MIMVS proved to be safe and ultimately effective. We hypothesize that avoiding general anesthesia for both TAVR and MIMVS reduced the patient's perioperative morbidity risks. ${ }^{4}$

Finally, it is important to consider other MIMVS techniques such as transapical mitral valve repair with NeoChord (St. Louis Park, Minnesota, United States) and transcatheter mitral valve replacement (TMVR) for high risk patients as presented in this case. NeoChord is currently under clinical trial and several devices for TMVR are under preclinical and clinical evaluations; however, these options were unavailable in Japan during this patient's treatment. Even if NeoChord and TMVR were available, the transapical approach would be the only option left postTAVR, requiring general anesthesia or the "awake" technique used in this case. Although the patient's MR was partially caused by torn chordae tendineae in P2, it is probable that treatment with NeoChord would have been insufficient because the mitral annulus dilation would have been forgone. In this case, TMVR would be a better choice, but placement of the mitral valve without disrupting the TAVR valve is risky and requires a high level of control and technical skill that comes with ample experience. Once transcatheter mitral valve techniques are available globally, we look forward to exploring those new treatment options.

\section{Conclusion}

For high-risk patients with multiple VHD such as comorbid AS and MR, MIMVS is a viable option when additional surgical treatment for MR is necessary post-TAVR.

Conflict of Interest

None declared.

\section{References}

1 Mueller XM, Tevaearai HT, Stumpe F, et al. Long-term results of mitral-aortic valve operations. J Thorac Cardiovasc Surg 1998;115 (06):1298-1309

2 Mavromatis K, Thourani VH, Stebbins A, et al. T AVR in patients with AS and MR. Ann Thorac Surg 2017;104:1977-1986

3 Sannino A, Grayburn PA. Mitral regurgitation in patients with severe aortic stenosis: diagnosis and management. Heart 2018; 104(01):16-22

4 Kanda H, Kamiya H, Sugawara A, et al. Minimally invasive awake mitral valve surgery and cardiopulmonary bypass without general anesthesia. Ann Thorac Surg 2019;107(04):e247-e248 